\title{
In vivo activity of anprocide alone, and in vitro activity in combination with conventional antibiotics against Staphylococcus aureus and Staphylococcus epidermidis biofilms
}

Correspondence

Robin K. Pettit

robin.pettit@asu.edu

Received 25 November 2008

Accepted 27 May 2009

\author{
Robin K. Pettit, ${ }^{1}$ Christine A. Weber, ${ }^{1} \dagger$ Stacey B. Lawrence, ${ }^{1}$ \\ George R. Pettit, ${ }^{1}$ Melissa J. Kean ${ }^{1}$ and Gary D. Cage ${ }^{2}$ \\ ${ }^{1}$ Cancer Research Institute and Department of Chemistry and Biochemistry, Arizona State \\ University, Tempe, AZ 85287, USA \\ ${ }^{2}$ Phoenix Children's Hospital, Phoenix, AZ 85016, USA
}

\begin{abstract}
The alarming spread of multiple drug resistance in Staphylococcus aureus, combined with the frequent occurrence of $S$. aureus and Staphylococcus epidermidis in biofilm-type infections, indicates a growing need for new therapies. The experimental steroidal amide anprocide [3 $\beta$-acetoxy-17 $\beta$-(L-prolyl)amino- $5 \alpha$-androstane] significantly reduced c.f.u. $\mathrm{ml}^{-1}$ per suture $(P<0.0001)$ in a murine model of topical $S$. aureus infection. In chequerboard assays with planktonic-grown $S$. aureus and $S$. epidermidis, anprocide was synergistic with bacitracin, oxacillin, clindamycin or ceftriaxone. Anprocide was also synergistic in combination with bacitracin or oxacillin against some isolates of biofilm-grown S. aureus and S. epidermidis.
\end{abstract}

\section{INTRODUCTION}

Antibiotic-resistant Staphylococcus aureus is a major public health problem. Meticillin-resistant $S$. aureus (MRSA) is increasing in both nosocomial and community-acquired infections (Lipsky et al., 2007). A 2004 National Nosocomial Infections Surveillance System Report (NNIS System, 2004) indicated that nearly $60 \%$ of intensive care unit $S$. aureus isolates were resistant to meticillin, oxacillin or nafcillin. There were 94360 invasive MRSA infections in the US in 2005, which were associated with death in 18650 cases (Klevens et al., 2007).

In the US, one million nosocomial infections each year are related to infections caused by biofilms on implanted devices (Schierholz \& Beuth, 2001). Mortality for septicaemias associated with vascular devices ranges from 20 to $40 \%$ (Stamm, 1978), and intravenous catheters are the most common cause of nosocomial septicaemia (Maki, 1992). S. aureus and Staphylococcus epidermidis are the most common infectious agents associated with foreign device infections (Moreillon \& Que, 2004; Waldvogel \& Bisno, 2000; Zimmerli et al., 2004), and are found in biofilms in a wide range of other diseases, including endocarditis and osteomyelitis (Donlan \& Costerton,

†Present address: Department of Pharmacy, Ludwig Maximilians University, Munich, Germany.

Abbreviations: AB, Alamar blue; FIC, fractional inhibitory concentration; MRSA, meticillin-resistant $S$. aureus.
2002). Bacterial biofilms are generally quite resistant to antibiotic treatment (Donlan \& Costerton, 2002).

The steroidal amide anprocide [ $3 \beta$-acetoxy- $17 \beta$-(L-prolyl)amino- $5 \alpha$-androstane] was synthesized in 1967 (Pettit et al., 1967). In 2000, its activity against 179 Gram-positive clinical isolates was reported (Pettit et al., 2000). Anprocide was bactericidal for the majority of MRSA, vancomycinresistant Enterococcus spp., penicillin-resistant Streptococcus pneumoniae, invasive Streptococcus pneumoniae, group A Streptococcus and Rhodococcus spp. isolates, and has activity against biofilms of S. epidermidis ATCC 35984 (Pettit et al., 2005). The maximum tolerated dose in mice is $>400 \mathrm{mg}$ $\mathrm{kg}^{-1}$ i.p. (Pettit et al., 2000). In the present report, the efficacy of anprocide in a murine model of topical S. aureus infection was investigated, as were possible synergistic interactions with approved antibiotics against planktonicand biofilm-grown S. aureus and S. epidermidis.

\section{METHODS}

Murine model. The animal study was performed at the Southern Research Institute, Frederick, MD, USA, with strict adherence to ethical standards. The mouse surgical wound infected with $S$. aureus model was used (Gisby \& Bryant, 2000). The murine model of skin wound infection represents the secondary skin infections that may occur following damage by accidental trauma, surgery and burns, or as a result of superinfection of an underlying skin disease (Gisby \& Bryant, 2000). Mouse virulent S. aureus ATCC 14154 was grown for $12 \mathrm{~h}$ at $38{ }^{\circ} \mathrm{C}$ in brain heart infusion broth. Just prior to surgery, sterile silk sutures were cut into $1 \mathrm{~cm}$ lengths, soaked in the undiluted 
broth culture for $30 \mathrm{~min}$ and excess liquid was removed by blotting with sterile filter paper. Fifty HSD : ICR mice (five groups, 10 animals each) were anaesthetized and the skin on their backs was prepared for aseptic surgery by clipping, cleansing with iodine scrub and rinsing with alcohol. Mice were kept warm during surgery on a recirculating hot water blanket. Sutures were placed just under the skin on the dorsum of each mouse using sterile instruments. Sutures were knotted in the subcutaneous tissue to keep them in place. A halfthickness skin wound was made over the sutures using the side of an 18 gauge needle as a scalpel. Treatment was initiated $4 \mathrm{~h}$ after surgery. Anprocide was reconstituted in $\mathrm{MeOH} /$ saline and placed over the wound site twice daily at 12.5 or $25 \mathrm{mg} \mathrm{kg}^{-1}$ per day. Enrofloxacin (Baytril) was given subcutaneously once a day at $10 \mathrm{mg} \mathrm{kg}^{-1}$ per day. After 7 days of treatment, mice were euthanized with i.p. pentobarbital solution. Two groups of mice were untreated and served as controls: one had infected sutures and the other sterile sutures. Sutures were aseptically removed, vortexed in PBS, and Staphylococcus c.f.u. per suture were determined by dilution plating. The Mann-Whitney nonparametric $t$-test was used to determine statistical significance.

Planktonic chequerboard assay. Anprocide was tested alone and in combination with seven antibiotics against clinical isolates and type strains of S. aureus and S. epidermidis. Nonduplicate clinical isolates (from sterile sites) and antibiotic resistance information were obtained from the Arizona Department of Health Services and Phoenix Children's Hospital (identified to species level using the Vitek II system). Reference strains were obtained from the American Type Culture Collection (ATCC). For S. aureus planktonic chequerboard assays, nine meticillin-resistant clinical isolates and ATCC 29213 were used. For S. epidermidis planktonic chequerboard assays, seven clinical isolates and ATCC 35984, ATCC 49461 and ATCC 12228 were used. Bacitracin, vancomycin, nitrofurazone, enrofloxacin and clindamycin were obtained from ICN. Ceftriaxone and oxacillin were from Sigma. Nitrofurazone, enrofloxacin and clindamycin were dissolved in sterile DMSO and the remaining antibiotics were dissolved in sterile $\mathrm{H}_{2} \mathrm{O}$. Anprocide was synthesized in our laboratory as previously described (Pettit et al., 1967), and prior to each assay, dissolved in $\mathrm{MeOH}$. Planktonic susceptibility testing of S. aureus and $S$. epidermidis was performed by the reference broth microdilution assay outlined by the Clinical and Laboratory Standards Institute (CLSI) (NCCLS, 2000), using round-bottom, polystyrene, tissue culture treated microtitre plates and cation-adjusted Mueller-Hinton II broth. Turbidity controls containing the highest concentrations of DMSO or $\mathrm{MeOH}$ were identical in appearance to turbidity controls lacking solvent. The MIC was defined as the lowest concentration of drug that inhibited all visible growth of the test organism (optically clear). Planktonic chequerboard assays were performed using a wellestablished method (Eliopoulos \& Moellering, 1996). Drug interaction was classified as synergistic, indifferent or antagonistic on the basis of the fractional inhibitory concentration (FIC) index. The FIC index is the sum of the FIC of each of the drugs, which in turn is defined as the MIC of each drug when used in combination divided by the MIC of the drug when used alone. The interaction was defined as synergistic if the FIC index was less than or equal to 0.5, indifferent if the FIC index was greater than 0.5 and less than or equal to 4.0 , and antagonistic if the FIC index was greater than 4.0 (Barchiesi et al., 2004).

Biofilm chequerboard assay. Four biofilm-positive isolates (confirmed with Congo red-stained phase-contrast micrographs) were used for each species (Pettit et al., 2005; R. K. Pettit, C. A. Weber \& G. R. Pettit, unpublished): S. aureus ATCC 29213 and clinical isolates (from sterile sites) 520009, 520016 and 520020 (Arizona Department of Health Services, Phoenix, AZ, USA), and S. epidermidis ATCC 12228, ATCC 35984 (RP62A) and two clinical isolates, S67166 (central venous catheter) and X64787 (endotracheal tube) (Phoenix
Children's Hospital, Phoenix, AZ, USA). S. epidermidis ATCC 12228 is sometimes referred to as biofilm-negative, perhaps because the biofilms formed are not as robust as those of biofilm-positive strain ATCC 35984. However, S. epidermidis ATCC 12228 has been shown to produce biofilms in many studies (Greco et al., 2007; Henriques et al., 2005; Okajima et al., 2006; Pettit et al., 2005), and may provide another example of biofilm formation via ica-independent means (Greco et al., 2007; Kogan et al., 2006; Rohde et al., 2005). The method used to prepare and drug treat biofilms was exactly as previously described (Pettit et al., 2005; R. K. Pettit, C. A. Weber \& G. R. Pettit, unpublished), except that drug combinations instead of individual drugs were used. Addition of Alamar blue (AB) and calculation of per cent reduction was exactly as previously described (Pettit et al., 2005). FIC indices were calculated as described above.

\section{RESULTS AND DISCUSSION}

With the continuing increase in resistance of Staphylococcus to available antimicrobials, there is a pressing need for novel compounds and effective drug combinations. In a murine surgical wound model, anprocide caused a 500010000 -fold reduction in c.f.u. per suture (Table 1), with no weight loss or other adverse clinical effects noted. There was some difficulty in keeping the anprocide in contact with the wound. As such, anprocide may be even more efficacious when in an ointment or cream formulation or on an adhesive bandage.

Antimicrobial combinations may increase the rate of microbial killing, shorten the duration of therapy, avoid the emergence of drug resistance, expand the spectrum of activity and decrease drug-related toxicities by permitting use of lower doses (Barchiesi et al., 2001). Anprocide was synergistic in combination with bacitracin, oxacillin, clindamycin and ceftriaxone for some isolates of $S$. aureus, and synergistic with bacitracin, oxacillin, clindamycin, ceftriaxone and vancomycin for some isolates of $S$. epidermidis (Table 2). None of the tested combinations were antagonistic.

S. aureus and $S$. epidermidis are the most common infectious agents associated with foreign device infections (Moreillon \& Que, 2004; Waldvogel \& Bisno, 2000; Zimmerli et al., 2004), and are found in biofilms in a wide range of other diseases, including endocarditis and

Table 1. Efficacy of anprocide in a murine model of topical $S$. aureus infection

\begin{tabular}{|lccc|}
\hline Treatment & $\begin{array}{c}\text { Dose }\left(\mathbf{m g ~ k g}^{-\mathbf{1}}\right. \\
\text { per day) }\end{array}$ & $\begin{array}{c}\text { Log c.f.u. } \\
\text { per suture } \\
(\mathbf{m} \text { ean } \mathbf{l o g} \pm \text { SD) }\end{array}$ & $\begin{array}{c}\boldsymbol{P} \text {-value vs } \\
\text { no therapy }\end{array}$ \\
\hline Anprocide & 12.5 & $2.90 \pm 0.33$ & $<0.0001$ \\
Anprocide & 25 & $2.66 \pm 0.15$ & $<0.0001$ \\
Baytril & 10 & $1.33 \pm 0.86$ & $<0.0001$ \\
Untreated & & $6.54 \pm 0.37$ & \\
$\begin{array}{l}\text { No infection, no } \\
\text { treatment }\end{array}$ & & 0 & \\
\hline
\end{tabular}


Table 2. In vitro activities of anprocide in combination with conventional antibiotics against planktonic-grown $S$. aureus and $S$. epidermidis

\begin{tabular}{|lcc|}
\hline $\begin{array}{l}\text { Drug used in } \\
\text { combination } \\
\text { with anprocide }\end{array}$ & $\begin{array}{l}\text { Percentage of isolates where results } \\
\text { were synergistic (FIC index } \leqslant \mathbf{0 . 5})^{\star}\end{array}$ \\
\cline { 2 - 3 } & $\begin{array}{c}\text { S. } \text { aureus } \\
(\boldsymbol{n}=\mathbf{1 0} \text { isolates })\end{array}$ & $\begin{array}{c}\text { S. } \text { epidermidis } \\
(\boldsymbol{n}=\mathbf{1 0} \text { isolates })\end{array}$ \\
\hline Nitrofurazone & 0 & 0 \\
Bacitracin & 40 & 30 \\
Oxacillin & 10 & 50 \\
Clindamycin & 90 & 10 \\
Ceftriaxone & 40 & 10 \\
Enrofloxacin & 0 & 0 \\
Vancomycin & 0 & 10 \\
\hline
\end{tabular}

${ }^{\star}$ Results for remaining strains were indifferent (FIC index $>0.5-4.0$ ).

osteomyelitis (Donlan \& Costerton, 2002). Two drug combinations that were particularly effective against either $S$. aureus or $S$. epidermidis grown planktonically were evaluated against biofilms using the microplate $A B$ biofilm susceptibility assay (Pettit et al., 2005; R. K. Pettit, C. A. Weber \& G. R. Pettit, unpublished). The AB method has excellent correlation with c.f.u. $\mathrm{ml}^{-1}$ (Pettit et al., 2005); as such, the $A B$ biofilm susceptibility assay provides a simple way of assessing biofilm viability.

When S. aureus was grown planktonically, the combination of anprocide and bacitracin was synergistic for clinical isolate 520020 and indifferent for ATCC 29213 and clinical isolates 520009 and 520016. When S. epidermidis was grown planktonically, the combination of anprocide and bacitracin was synergistic for ATCC 35984 and clinical isolate X64787, and indifferent for ATCC 12228 and clinical isolate S67166. When grown as biofilms, the combination of anprocide and bacitracin was synergistic for all four isolates of S. epidermidis and 2/4 (except for one replicate with ATCC 29213) isolates of S. aureus (Table 3). The combination of anprocide and oxacillin was synergistic for S. aureus clinical isolate 520016 when grown planktonically, and indifferent for ATCC 29213 and clinical isolates 520009 and 520020 when grown planktonically. The combination of anprocide and oxacillin was synergistic for S. epidermidis clinical isolates S67166 and X64787 when grown planktonically, and indifferent for ATCC 12228 and ATCC 35984 when grown planktonically. For biofilms of $S$. aureus, the combination of anprocide and oxacillin was indifferent (except for one replicate with clinical isolate 520016) (Table 3). For biofilms of S. epidermidis, the combination of anprocide and oxacillin was synergistic in just over half of the replicate tests (Table 3). Although clindamycin and anprocide were synergistic for 9/10 $\mathrm{S}$. aureus isolates grown planktonically (Table 2), the combination was indifferent when evaluated against the four isolates of $S$. aureus grown as biofilms (data not shown).

As demonstrated, the $\mathrm{AB}$ biofilm susceptibility method works well for drug combination studies as well as single drug studies using $S$. aureus and $S$. epidermidis. We previously reported (Pettit et al., 2005) that none of eight FDA-approved antibiotics inhibited S. epidermidis ATCC 35984 biofilms at $<512 \mu \mathrm{g} \mathrm{ml}{ }^{-1}$ [most, including bacitracin and oxacillin, had minimum biofilm inhibitory concentrations (MBICs) $>4096 \mu \mathrm{g} \mathrm{ml}^{-1}$. As shown in the current study, there was a dramatic reduction in the MBICs of bacitracin and oxacillin in the presence of anprocide (e.g. for S. epidermidis, the MBIC of bacitracin $=16384 \mu \mathrm{g}$ $\mathrm{ml}^{-1}$, while the MBIC of bacitracin + anprocide $=4-8 \mu \mathrm{g}$ $\mathrm{ml}^{-1}$ ), suggesting that these drugs have different mechanisms/targets which are mutually beneficial to the antibacterial action. The mechanism by which one drug enhances the activity of the other was not investigated here. Given that anprocide is a steroid, it may be membrane-active, and could facilitate the uptake of bacitracin and oxacillin.

Anprocide should be pursued as a possible treatment for Gram-positive infections. Anprocide is unrelated to systemically administered agents, well tolerated at high doses (Pettit et al., 2000), has a spontaneous mutation rate in the expected range for a compound in early preclinical

Table 3. Effect of anprocide in combination with bacitracin or oxacillin on S. aureus and S. epidermidis biofilms

\begin{tabular}{|lcccc|}
\hline Strain & $\begin{array}{c}\text { Range of FIC index } \\
\text { anprocide/bacitracin }\end{array}$ & $\begin{array}{c}\text { No. replicates exhibiting } \\
\text { synergy }\end{array}$ & $\begin{array}{c}\text { Range of FIC index } \begin{array}{c}\text { replicates } \\
\text { anprocide/oxacillin }\end{array} \\
\text { synergy }{ }^{\star} / \text { no. replicates }\end{array}$ \\
\hline S. aureus ATCC 29213 & $0.14-1.00$ & $2 / 3$ & $2.00-3.00$ & $0 / 2$ \\
S. aureus 520009 & $0.14-0.15$ & $2 / 2$ & 2.00 & $0 / 2$ \\
S. aureus 520016 & $0.51-0.56$ & $0 / 2$ & $0.37-0.53$ & $1 / 2$ \\
S. aureus 520020 & 2.00 & $0 / 2$ & 2.00 & $0 / 2$ \\
S. epidermidis ATCC 35984 & $0.25-0.50$ & $4 / 4$ & $0.50-0.53$ & $2 / 4$ \\
S. epidermidis ATCC 12228 & $0.09-0.50$ & $4 / 4$ & $0.28-1.00$ & $3 / 4$ \\
S. epidermidis S67166 & $0.25-0.50$ & $4 / 4$ & $0.50-1.00$ & $2 / 4$ \\
S. epidermidis X64787 & $0.25-0.50$ & $4 / 4$ & $0.50-1.00$ & $1 / 2$ \\
\hline
\end{tabular}

${ }^{\star}$ Remaining replicates exhibited indifference. 
development (Pettit et al., 2000), is active at pH 6-8 (Pettit et al., 2000), bactericidal (Pettit et al., 2000), and has now been shown to have topical in vivo activity, and in vitro activity against biofilms alone (Pettit et al., 2005), and in combination with bacitracin.

\section{ACKNOWLEDGEMENTS}

Financial support was provided by grants R01 CA90441-01-05, 2R56 CA090441-06A1 and 5R01 CA090441-07 from the Division of Cancer Treatment, Diagnosis and Centers, National Cancer Institute, DHHS, the Arizona Biomedical Research Commission, the Robert B. Dalton Endowment Fund and Dr Alec Keith.

\section{REFERENCES}

Barchiesi, F., Schimizzi, A. M., Najvar, L. K., Bocanegra, R., Caselli, F., Di Cesare, S., Giannini, D., Di Francesco, L. F., Giacometti, A. \& other authors (2001). Interactions of posaconazole and flucytosine against Cryptococcus neoformans. Antimicrob Agents Chemother 45, 1355-1359.

Barchiesi, F., Spreghini, E., Maracci, M., Fothergill, A. W., Baldassarri, I., Rinaldi, M. G. \& Scalise, G. (2004). In vitro activities of voriconazole in combination with three other antifungal agents against Candida albicans. Antimicrob Agents Chemother 48, 3317-3322.

Donlan, R. M. \& Costerton, J. W. (2002). Biofilms: survival mechanisms of clinically relevant microorganisms. Clin Microbiol Rev 15, 167-193.

Eliopoulos, G. M. \& Moellering, R. C., Jr (1996). Antimicrobial combinations. In Antibiotics in Laboratory Medicine, 4th edn, pp. 330396. Edited by V. Lorian. Baltimore, MD: Williams \& Wilkins.

Gisby, J. \& Bryant, J. (2000). Efficacy of a new cream formulation of mupirocin: comparison with oral and topical agents in experimental skin infections. Antimicrob Agents Chemother 44, 255-260.

Greco, C., Martincic, I., Gusinjac, A., Kalab, M., Yang, A. F. \& Ramírez-Arcos, S. (2007). Staphylococcus epidermidis forms biofilms under simulated platelet storage conditions. Transfusion 47, 11431153.

Henriques, M., Sousa, C., Lira, M., Elisabete, M., Oliveira, R., Oliveira, R. \& Azeredo, J. (2005). Adhesion of Pseudomonas aeruginosa and Staphylococcus epidermidis to silicone-hydrogel contact lenses. Optom Vis Sci 82, 446-450.

Klevens, R. M., Morrison, M. A., Nadle, J., Petit, S., Gershman, K., Ray, S., Harrison, L. H., Lynfield, R., Dumyati, G. \& other authors (2007). Invasive methicillin-resistant Staphylococcus aureus infections in the United States. JAMA 298, 1763-1771.
Kogan, G., Sadovskaya, I., Chaignon, P., Chokr, A. \& Jabbouri, S. (2006). Biofilms of clinical strains of Staphylococcus that do not contain polysaccharide intercellular adhesin. FEMS Microbiol Lett 255, 11-16.

Lipsky, B. A., Weigelt, J. A., Gupta, V., Killian, A. \& Peng, M. M. (2007). Skin, soft tissue, bone, and joint infections in hospitalized patients: epidemiology and microbiological, clinical, and economic outcomes. Infect Control Hosp Epidemiol 28, 1290-1298.

Maki, D. G. (1992). Infections due to infusion therapy. In Hospital Infections, pp. 849-898. Edited by J. V. Bennett \& P. S. Brachman. Boston, MA: Little, Brown \& Co.

Moreillon, P. \& Que, Y. A. (2004). Infective endocarditis. Lancet 363, 139-149.

NCCLS (2000). Methods for Dilution Antimicrobial Susceptibility Tests for Bacteria that grow Aerobically; Approved Standard M7-A5. Wayne, PA: National Committee for Clinical Laboratory Standards.

NNIS System (2004). National Nosocomial Infections Surveillance (NNIS) System Report, data summary from January 1992 through June 2004, issued October 2004. Am J Infect Control 32, 470-485.

Okajima, Y., Kobayakawa, S., Tsuji, A. \& Tochikubo, T. (2006). Biofilm formation by Staphylococcus epidermidis on intraocular lens material. Invest Ophthalmol Vis Sci 47, 2971-2975.

Pettit, G. R., Smith, R. L. \& Klinger, H. (1967). Synthesis of $3 \beta$-acetoxy$17 \beta$-(L-arginyl-L-arginyl-L-prolyl)amino- $5 \alpha$-androstane. J Med Chem 10, 145-148.

Pettit, R. K., Cage, G. D., Pettit, G. R. \& Liebman, J. A. (2000). Antimicrobial and cancer cell growth inhibitory activities of $3 \beta$ acetoxy- $17 \beta$-(L-prolyl)amino- $5 \alpha$-androstane in vitro. Int J Antimicrob Agents 15, 299-304.

Pettit, R. K., Weber, C. A., Kean, M. J., Hoffmann, H., Pettit, G. R., Tan, R., Franks, K. S. \& Horton, M. L. (2005). Microplate alamar blue assay for Staphylococcus epidermidis biofilm susceptibility testing. Antimicrob Agents Chemother 49, 2612-2617.

Rohde, H., Burdelski, C., Bartscht, K., Hussain, M., Buck, F., Horstkotte, M. A., Knobloch, J. K. M., Heilmann, C., Herrmann, M. \& Mack, D. (2005). Induction of Staphylococcus epidermidis biofilm formation via proteolytic processing of the accumulation-associated protein by staphylococcal and host proteases. Mol Microbiol 55, 18831895.

Schierholz, J. M. \& Beuth, J. (2001). Implant infections: a haven for opportunistic bacteria. J Hosp Infect 49, 87-93.

Stamm, W. E. (1978). Prevention of infections. Infections related to medical devices. Ann Intern Med 89, 764-769.

Waldvogel, F. A. \& Bisno, A. L. (2000). Infections Associated with Indwelling Medical Devices, 3rd edn. Washington, DC: American Society for Microbiology.

Zimmerli, W., Trampuz, A. \& Ochsner, P. E. (2004). Prosthetic-joint infections. N Engl J Med 351, 1645-1654. 